\title{
Charge state dependent top-down characterisation using electron transfer dissociation
}

\author{
Marko Rožman and Simon J. Gaskell
}

The dissociation of protein ions $(5-30 \mathrm{kDa})$ as a function of charge state has been explored in order to suggest the optimal charge state range for top-down sequencing. Proteins were generated under denaturing conditions and their charge states were modified via ion/ion proton transfer reactions prior to dissociation. Electron transfer dissociation (ETD) data suggested optimal sequence coverage for charge states in the $\mathrm{m} / \mathrm{z}$ range from 700 to 950 while limited sequence coverage was noted when the precursor $\mathrm{m} / \mathrm{z}$ was above 1000. Sequence coverage from ETD data was found to be dependent on protein size, with smaller proteins having better sequence coverage. An observed depletion in sequence-related information was mainly attributed to limited instrument (ion trap) performance ( $\mathrm{m} / \mathrm{z}$ range and resolution). For a combined ETD/collision-induced dissociation (CID) approach it is difficult to propose an optimal $\mathrm{m} / \mathrm{z}$ range since good sequence coverage for CID is at intermediate charge states and the optimal $\mathrm{m} / \mathrm{z}$ range increases with protein size. When only one charge state can be analysed in a combined ETD/CID approach, a range around $950 \mathrm{~m} / \mathrm{z}$ is suggested as a starting point. Alternatively, two charge states should be explored, each optimal for either ETD or CID. Overall, these suggestions should be useful to achieve enhanced characterisation of smaller proteins/large protein fragments (generated from denaturing solutions) in minimal analysis times.

Mass spectrometry (MS) has emerged as the most favoured method for rapid 'classification' (identification, characterisation, quantification) of proteins. There are two ways of tackling protein analysis by MS: the bottom-up and the top-down approaches. ${ }^{[1,2]}$ In the bottom-up strategy, proteins are subjected to proteolytic digestion and the resulting peptide mixture is analysed in the gas phase by MS and/or tandem mass spectrometry (MS/MS). On the other hand, the top-down approach uses intact proteins or large protein fragments (sometimes termed the 'middle-down' approach) which are introduced into the gas phase, fragmented and analysed by MS. At the moment, the majority of protein 'classification' is done by the bottom-up strategy. ${ }^{[2-4]}$ However, potential use of the intact proteins rather than proteolytic fragments can reduce the complexity of the analytical space and immediately provide the primary structure (rather than of a fragment) as well as reveal its potential modifications. So far, application of the top-down strategy has mostly focused on single purified proteins. ${ }^{[2-4]}$ It has been suggested that one of the improvements in top-down MS should be the ability to analyse large numbers of proteins when dealing with limited amounts of complex mixtures. ${ }^{[2-4]}$ Therefore, maximum structural information obtained in the shortest measurement time is desirable.
Proteins formed by electrospray ionisation (ESI) produce complex mass spectra with multiple charge states. When dealing with mixtures of proteins, spectra can become even more complex in terms of multiple overlaps between different charge states of different proteins in a narrow mass to charge range. Considering the limited time for MS/MS analysis (e.g. on-line high-throughput experiment) prediction of the charge state that gives the best sequence coverage and provides enough diagnostic information may be vital.

Different charge states of the same peptide or protein can have distinct fragmentation properties and yield different fragmentation product ions. ${ }^{[5,6]}$ Due to the use of denaturing solutions higher charge states tend to be formed by ESI. ${ }^{[7]}$ However, various charge state manipulations, e.g. ion/molecule and ion/ ion proton transfer reactions as well as metal cationisation, allow other charge states to be accessed. ${ }^{[5,6,8,9]}$ Charge state dependent behaviour of protein ions has been extensively studied by McLuckey's group, where multiply charged ions were charge manipulated before and after collision-induced dissociation (CID). ${ }^{[5,10-21]}$ These studies have suggested that, in the top-down approach, the dissociation channels are strongly dependent on precursor ion charge state. Low and high charge states show relatively modest sequence coverage while intermediate charge states yield more information. The low charge states favoured cleavage C-terminal to Asp residues as well as losses of small neutral molecules, while the highest charge states favoured cleavages N-terminal to Pro residue. Non-specific amide bond fragmentation was observed at the intermediate charge states.

Electron transfer/capture dissociation (ETD and ECD) is the second most widely used approach to induce protein ion fragmentation. ${ }^{[2,23]}$ It is an alternative and complementary 

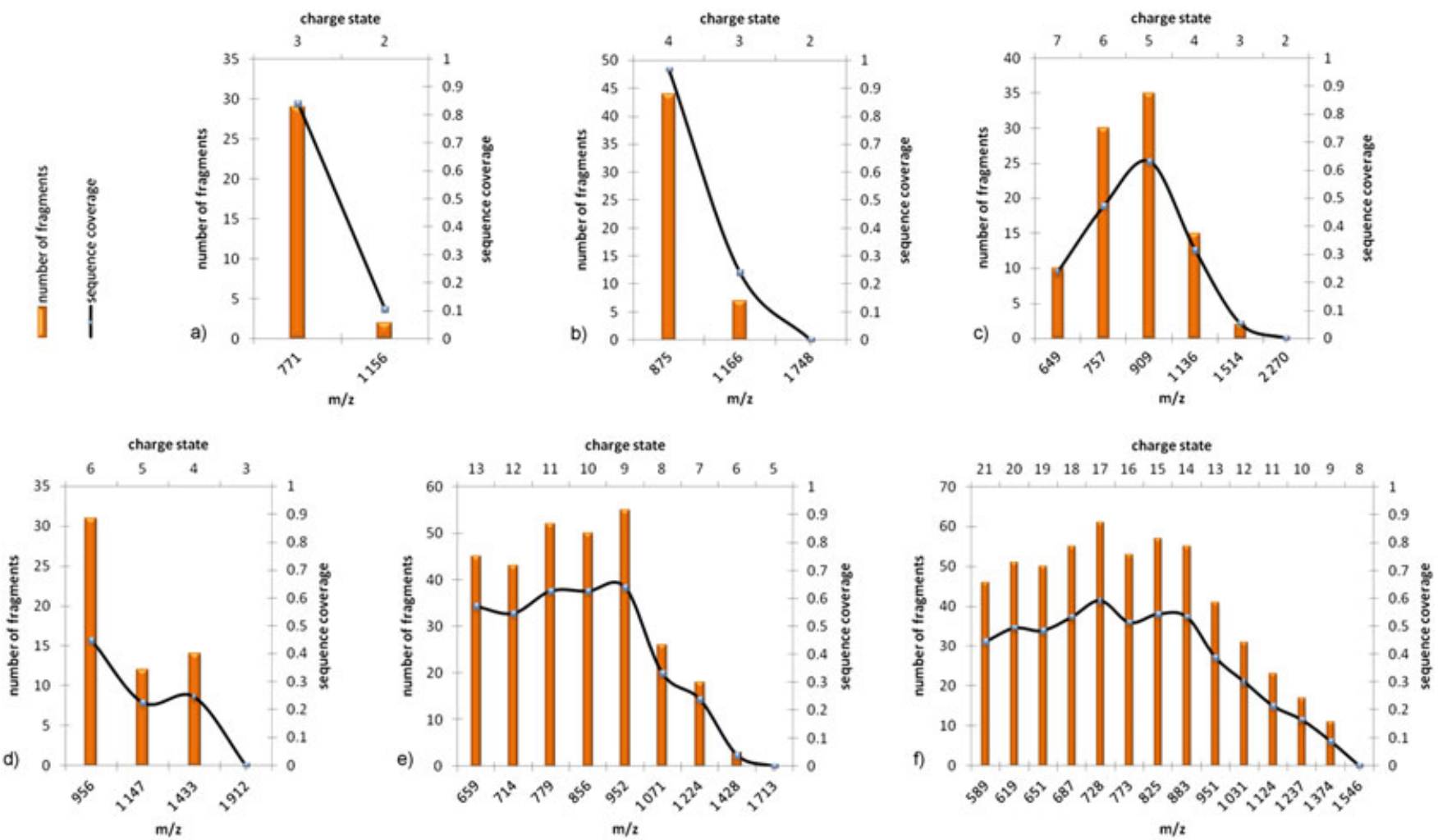

charge state
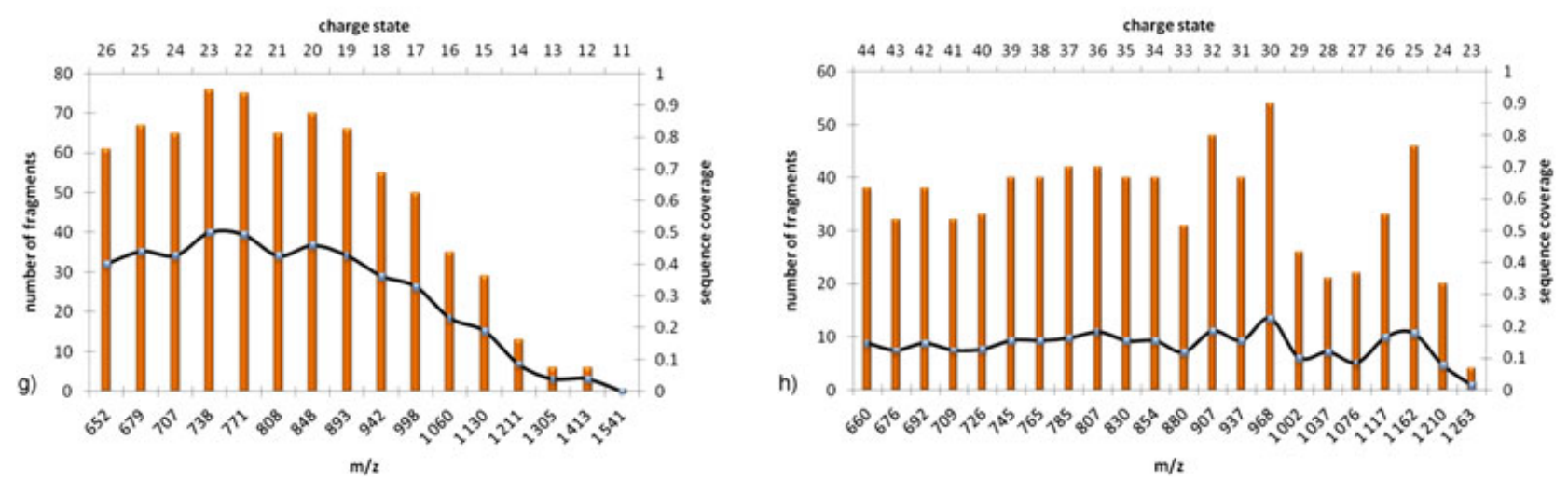

Figure 2. Number of product ions and sequence coverage generated by ETD as a function of precursor charge state: (a) tumor necrosis factor- $\alpha$ fragment $(2.3 \mathrm{kDa})$; (b) insulin $\beta$ chain $(3.5 \mathrm{kDa})$; (c) corticotropin A (4.5 kDa); (d) insulin (5.7 kDa); (e) ubiquitin ( $8.5 \mathrm{kDa}) ;(\mathrm{f})$ cytochrome C (12.4 kDa); (g) myoglobin (17 kDa); and (h) carbonic anhydrase (29 kDa).

coverage is observed. Maximum sequence coverage occurs at transition from high to intermediate charge state range. Intermediate charge states exhibit a sudden decrease of $\sim 10 \%$ in sequence coverage and at the transition from intermediate to low charge states dissociation usually becomes highly inefficient. A more general picture is obtained when an $m / z$ scale is used (Fig. 2). Good to maximum sequence coverage is obtained at the precursor range of $m / z 700$ to 950 and a sharp coverage decrease happens when the precursor $m / z$ is over 1000. It follows for unknown proteins analysed by ETD, a precursor charge state in a range $\mathrm{m} / \mathrm{z} 700-900$ would likely give the best sequence coverage.

Moving from smaller toward larger proteins a decrease in the sequence coverage was observed. Our data set starts from $90 \%$ sequence coverage for e.g. insulin chain B, while for carbonic anhydrase (the largest protein analysed) sequence coverage reduces to $22 \%$. Figure 3 represents sequence

\section{SHJHJWJGJYJGJKJHJNJGPJEJHJWJHJKJDJFPIJAJN JGJEJRJQSPJVJDJIDJTJKJAWJ JUJPPAJLKPLALVJYJ GJEJATSRRMVNNGHSFNVEYDDSQDKAVLKDGPLT GTYRLVQFHFHWGSSDDQGSEHTVDRKKYAAELHL VHWNTKYGDFGTAAQQPDGLAVVGVLKVGDANPA LQKVLDALDSIKTKGKSTDFPNFDPGSLLPNVLDYWT

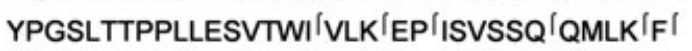

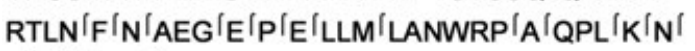 RIQ'VIRG'FPK}

Figure 3. Sequence coverage for carbonic anhydrase charge state $30+$ generated by ETD.

coverage for carbonic anhydrase charge state 30+. However, much better coverage (65\%) for the first and last 35 amino acids is obtained. This observation is likely associated with 
the upper $m / z$ limit of the HCT ion trap instrument $(3000 \mathrm{~m} / \mathrm{z}$ ). Post-dissociation PTR can place larger fragments outside of the $m / z$ range of the instrument. ${ }^{[21]}$ On the other hand, due to the limited resolution $(\sim 0.30 \mathrm{u}$ FWHM), multiply charged fragments without PTR cannot be resolved. Thus there are limitations on sequencing the interior region of the larger proteins. Although it has been reported that larger proteins exhibit much stronger electrostatic intramolecular interactions which can lead to intractable structure cleavable only at its ends, ${ }^{[25]}$ we believe that the main reason for limited sequence coverage in the current study is related to instrument performance. In terms of protein identification, good sequence coverage of the $\mathrm{N}$ - and $\mathrm{C}$-terminus obtained by top-down ETD has still the advantage over standard bottom-up analysis because ion mass values need only be matched against DNA terminal fragments instead of the whole sequence. ${ }^{[25]}$

\section{Combination of ETD and CID}

The use of alternative ion activation methods can improve analyte identification and enhance confidence in its recognition. ETD and CID are complementary dissociation methods and it is useful to obtain data from both approaches. ${ }^{[21,26]}$

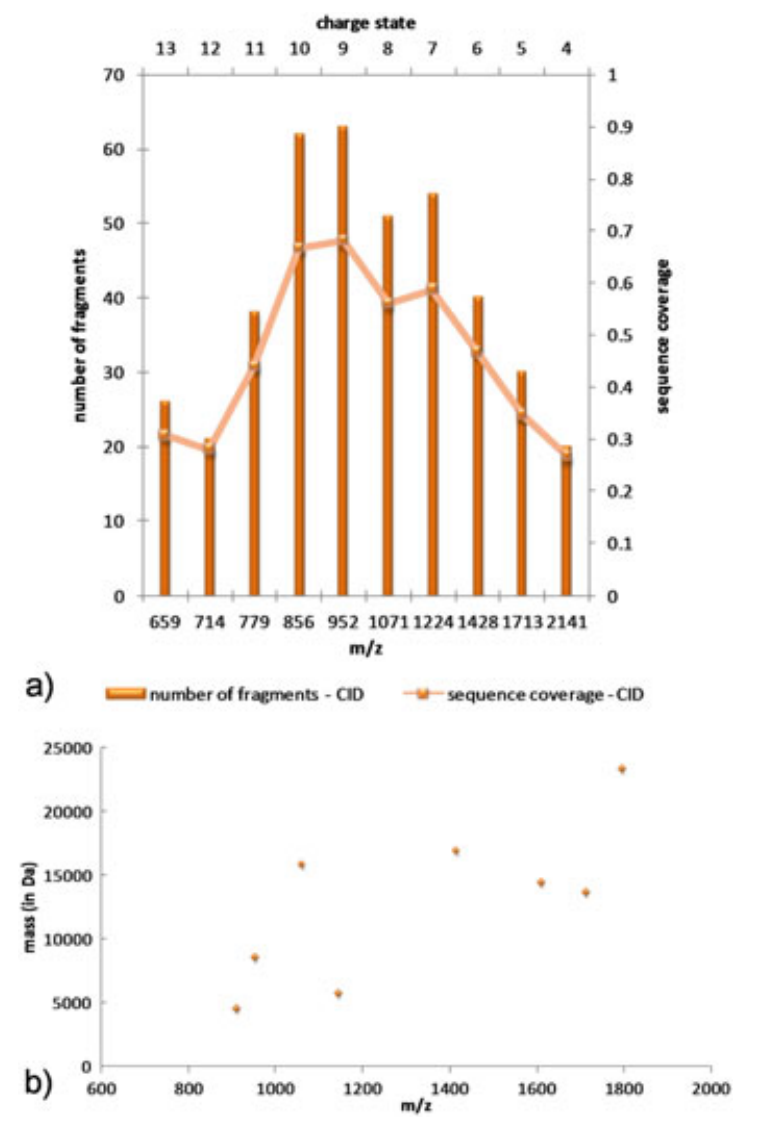

Figure 4. (a) Number of product ions and sequence coverage generated by CID for ubiquitin as a function of precursor charge state. (b) The best fragmenting charge state under CID conditions of a several proteins plotted on $\mathrm{m} / \mathrm{z}$ scale: $4.5 \mathrm{kDa}-$ corticotropin A; $5.7 \mathrm{kDa}$ - bovine insulin; ${ }^{[12]} 8.5 \mathrm{kDa}$ - ubiquitin; $13.7 \mathrm{kDa}-$ ribonuclease $\mathrm{A}_{i}^{[15]} 14.5 \mathrm{kDa}-\alpha$-synuclein; $[20]$ $15.7 \mathrm{kDa}$ - hemoglobin $\beta$-chain;; ${ }^{[11]} 17 \mathrm{kDa}$ - apomyoglobin; ${ }^{[13]}$ $23.3 \mathrm{kDa}$ - bovine trypsin. ${ }^{[19]}$
Sequence coverage obtained with charge state dependent top-down CID has a shape of an open down parabolic curve (Fig. 4(a)). Low and high charge states show relatively modest sequence coverage while intermediate charge states provide, in accordance with previous observations, the best sequence coverage. ${ }^{[5,10-21]}$ The best fragmenting (under CID) charge state of several proteins (estimated from literature ${ }^{[11-13,15,19,20]}$ ) plotted on the $m / z$ scale suggests that as the mass of a protein increases, there is also an increase in $\mathrm{m} / \mathrm{z}$ of the best fragmenting precursor ion (Fig. 4(b)). The plot starts with $\sim 900 \mathrm{~m} / \mathrm{z}$ for the charge state of the smallest protein considered $(4.5 \mathrm{kDa})$. Since for ETD the best sequence coverage can be expected (on the basis of the results reported here) for charge states in a range from 700 to $950 \mathrm{~m} / \mathrm{z}$, there could be a problem if in a combined ETD/CID approach just one charge state can be analysed (e.g. short measurement time). In that case a good starting point could be a charge state which falls in the upper end of the $\mathrm{m} / \mathrm{z}$ range suggested for ETD approach. Otherwise, two charge states should be considered, each optimal for corresponding dissociating technique applied, i.e. one of the intermediate charge states for CID and one from the range of $\mathrm{m} / \mathrm{z} 700$ to 950 for ETD.

\section{CONCLUSIONS}

The charge state dependent ETD of several protein ions was investigated. Good to maximum sequence coverage is obtained for charge states in the $\mathrm{m} / \mathrm{z}$ range from 700 to 950 . Limited sequence coverage was observed when the precursor $\mathrm{m} / \mathrm{z}$ is over 1000 . Moving from smaller toward larger proteins a decrease in the sequence coverage was observed and is explained by limited instrument (ion trap) performance $(\mathrm{m} / \mathrm{z}$ range and resolution).

The charge state dependent ETD data were used to suggest an optimal charge state range for the top-down protein sequencing. The range from 700 to $950 \mathrm{~m} / \mathrm{z}$ is suggested for the ETD approach. For the combined ETD/CID approach, when only one charge state can be considered, the range around $950 \mathrm{~m} / \mathrm{z}$ is implied as a good starting point. Otherwise, two charge states should be explored, each optimal for decomposition process (ETD or CID). These general suggestions are based on dissociation behaviour of 5 to $30 \mathrm{kDa}$ peptide/protein ions electrosprayed under denaturing conditions and therefore should be mainly applicable for smaller proteins/large protein fragments in similar conditions and mass range.

\section{Acknowledgements}

The Ministry of Science, Education and Sports of Republic of Croatia (Grant Number: 098-0982915-2945) and The Engineering and Physical Sciences Research Council - UK (Grant Number: EP/D013615/1) supported this work.

\section{REFERENCES}

[1] N. L. Kelleher, H. Y. Lin, G. A. Valaskovic, D. J. Aaserud, E. K. Fridriksson, F. W. McLafferty. Top down versus bottom up protein characterization by tandem high-resolution mass spectrometry. J. Am. Chem. Soc. 1999, 121, 806. 
[2] B. T. Chait. Mass spectrometry: bottom-up or top-down? Science 2006, 314, 65.

[3] R. Aebersold, M. Mann. Mass spectrometry-based proteomics. Nature 2003, 422, 198

[4] B. F. Cravatt, G. M. Simon, J.R. Yates III. The biological impact of mass spectrometry based proteomics. Nature 2007, 450, 991.

[5] G. E. Reid, J. Wu, P. A. Chrisman, J. M. Wells, S. A. McLuckey. Charge-state-dependent sequence analysis of protonated ubiquitin ions via ion trap tandem mass spectrometry. Anal. Chem. 2001, 73, 3274.

[6] M. Rožman, A. Schneider, S. J. Gaskell. Proton transfer reactions for improved peptide characterisation. J. Mass Spectrom. 2011, 46, 529.

[7] E. W. Robinson, R. D. Leib, E. R. Williams. The role of conformation on electron capture dissociation of ubiquitin. J. Am. Soc. Mass Spectrom. 2006, 17, 1469.

[8] S. A. McLuckey, G. J. Van Berkel, G. L. Glish. Reactions of dimethylamine with multiply charged ions of cytochrome c. J. Am. Chem. Soc. 1990, 112, 5668.

[9] M. Rožman, S. J. Gaskell. Non-covalent interactions of alkali metal cations with singly charged tryptic peptides. J. Mass. Spectrom. 2010, 45, 1409 .

[10] J. L. Stephenson Jr, B. J. Cargile, S. A. McLuckey. Ion trap collisional activation of disulfide linkage intact and reduced multiply protonated polypeptides. Rapid Commun. Mass Spectrom. 1999, 13, 2040.

[11] T. G. Schaaff, B. J. Cargile, J. L. Stephenson Jr, S. A. McLuckey. Ion trap collisional activation of the $(\mathrm{M}+2 \mathrm{H})^{2+}-(\mathrm{M}+17 \mathrm{H})^{17+}$ ions of human hemoglobin $\beta$-chain. Anal. Chem. 2000, 72, 899.

[12] J. M. Wells, J. L. Stephenson Jr, S. A. McLuckey. Charge dependence of protonated insulin decompositions. Int. J. Mass Spectrom. 2000, 203, A1.

[13] K. A. Newton, P. A. Chrisman, J. M. Wells, G. E. Reid, S. A. McLuckey. Gaseous apomyoglobin ion dissociation in a quadrupole ion trap: $[\mathrm{M}+2 \mathrm{H}]^{2+}-[\mathrm{M}+21 \mathrm{H}]^{21+}$ Int. J. Mass Spectrom. 2001, 212, 359.

[14] B. J. Engel, P. Pan, G. E. Reid, J. M. Wells, S. A. McLuckey. Charge state dependent fragmentation of gaseous protein ions in a quadrupole ion trap: bovine ferri-, ferro-, and apo-cytochrome c. Int. J. Mass Spectrom. 2002, 219,171
[15] G. E. Reid, J. L. Stephenson Jr, S. A. McLuckey, Tandem mass spectrometry of ribonuclease A and B: N-linked glycosylation site analysis of whole protein ions. Anal. Chem. 2002, 74, 577.

[16] J. M. Hogan, S. A. McLuckey. Charge state dependent collision-induced dissociation of native and reduced porcine elastase. J. Mass Spectrom. 2003, 38, 245.

[17] K. A. Newton, S. J. Pitteri, M. Laskowski, S. A. McLuckey. Effects of single amino acid substitution on the collisioninduced dissociation of intact protein ions: turkey ovomucoid third domain. J. Proteome Res. 2004, 3, 1033.

[18] Y. Xia, X. Liang, S. A. McLuckey. Ion trap versus low-energy beam-type collision-induced dissociation of protonated ubiquitin ions. Anal. Chem. 2006, 78, 1218.

[19] D. J. Watson, S. A. McLuckey. Charge state dependent ion trap collision-induced dissociation of reduced bovine and porcine trypsin cations. Int. J. Mass Spectrom. 2006, 255-256, 53.

[20] C. Chanthamontri, J. Liu, S. A. McLuckey. Charge state dependent fragmentation of gaseous $\alpha$-synuclein cations via ion trap and beam-type collisional activation. Int. J. Mass Spectrom. 2009, 283, 9.

[21] T.-y. Huang, S. A. McLuckey. Top-down protein characterization facilitated by ion/ion reactions on a quadrupole/ time of flight platform. Proteomics 2010, 10, 3577.

[22] R. A. Zubarev, N. L. Kelleher, F. W. McLafferty. Electron capture dissociation of multiply charged protein cations. a nonergodic process. J. Am. Chem. Soc. 1998, 120, 3265.

[23] J. E. P. Syka, J. J. Coon, M. J. Schroeder, J. Shabanowitz, D. F. Hunt, Peptide and protein sequence analysis by electron transfer dissociation mass spectrometry. Proc. Natl. Acad. Sci. USA 2004, 101, 9528.

[24] J. Liu, H. P. Gunawardena, T.-y. Huang, S. A. McLuckey. Charge-dependent dissociation of insulin cations via ion/ ion electron transfer. Int. J. Mass Spectrom. 2008, 276, 160.

[25] X. Han, M. Jin, K. Breuker, F. W. McLafferty. Extending topdown mass spectrometry to proteins with masses greater than 200 kilodaltons. Science 2006, 314, 109.

[26] S. R. Hart, K. W. Lau, Z. Hao, R. Broadhead, N. Portman, A. Hühmer, K. Gull, P. G. McKean, S. J. Hubbard, S. J. Gaskell. Analysis of the trypanosome flagellar proteome using a combined electron transfer/collisionally activated dissociation strategy. J. Am. Soc. Mass Spectrom. 2009, 20, 167. 